\title{
Light-Emitting-Diodes based on ordered InGaN nanocolumns emitting in the blue, green and yellow spectral range
}

\author{
A Bengoechea-Encabo , S Albert , D Lopez-Romero, P Lefebvre \\ F Barbagini , A Torres-Pardo , J M Gonzalez-Calbet \\ M A Sanchez-Garcia and E Calleja
}

\begin{abstract}
The growth of ordered arrays of InGaN/GaN nanocolumnar light emitting diodes by molecular beam epitaxy, emitting in the blue $(441 \mathrm{~nm})$, green $(502 \mathrm{~nm})$, and yellow $(568 \mathrm{~nm})$ spectral range is reported. The device active region, consisting of a nanocolumnar InGaN section of nominally constant composition and 250 to $500 \mathrm{~nm}$ length, is free of extended defects, which is in strong contrast to InGaN (planar) layers of similar composition and thickness. Electroluminescence spectra show a very small blue shift with increasing current (almost negligible in the yellow device) and line widths slightly broader than those of state-of-the-art InGaN quantum wells.
\end{abstract}

Keywords: InGaN, nanocolumns, LED, SAG

\section{Introduction}

The potential of InGaN alloys to generate light emission in the UV to IR range makes them an ideal choice for light emitting diodes (LED) covering the whole visible range and beyond. Typically, LEDs are based on multi quantum well (MQW) structures reaching their maximum external quantum efficiency (EQE) at relatively low current injection, below $10 \mathrm{~A} \mathrm{~cm}^{-2}$. At higher injection currents, the so-called efficiency 'droop' is observed even under pulsed current conditions, where self-heating effects are avoided. Several mechanisms such as carrier overflow and Auger recombination have been suggested as possible reasons to explain the droop effect [1-3]. Auger recombination is proportional to the third power of the carrier density $\left(\mathrm{n}^{3}\right)$, indicating that its impact would be drastically reduced by increasing the active region volume of the device (lower carrier density). This approach has been proposed and pursued by some groups using a double-heterostructure InGaN active layer, thicker

5 These authors contributed equally to this work than a typical QW, but still in the range of 10 to $20 \mathrm{~nm}[4,5]$. In these cases, an increase in the EQE has been observed, with a peak value shifting to higher currents. However, this approach would only be of real use if the thicker InGaN layers have good enough quality, i.e. no dislocations, which can be partly achieved by using free-standing GaN templates [5]. Indeed, it has been shown that the use of these low-defect substrates to grow LEDs with standard InGaN MQW active regions shifts the maximum $\mathrm{EQE}$ position to higher currents [6]. On the other hand, since the inefficient confinement of electrons in the InGaN active region is one of the reasons for the current overflow, a thicker region could also contribute to reduce this phenomenon, although other approaches, such as the use of electron blocking layers [3, 7], are more widespread.

As an alternative to planar devices, proposals of LEDs based on arrays of self-assembled nanocolumns (NCs) with embedded InGaN quantum disks (QDisk) or dots (QDs) have been reported in past years [8-12]. The advantages of NCbased LEDs are twofold: i) higher light extraction efficiency (without need of complicated technological processes) 
exploiting the guiding properties inherent to these 'cylindrical' nanostructures [13], and ii) active layers with very high crystalline quality and reduced strain. However, self-assembled NCs have critical drawbacks, such as an intrinsic polychromaticity and a strong geometrical and electrical dispersion that greatly complicate processing and hamper efficiency [8, 14].

In order to address these issues, selective area growth (SAG) of III-nitride NCs by molecular beam epitaxy (MBE) has been developed in recent years, achieving highly homogeneous and controllable arrays of InGaN/GaN nanocolumnar heterostructures with either embedded InGaN QDisks [15] or long InGaN portions [16, 17]. Recently, LEDs based on ordered arrays of NCs with embedded MQW emitting in the green [18] and red [19] as well as with an InGaN section of $80 \mathrm{~nm}$ thickness emitting in the near infrared range [20] have been demonstrated.

In contrast, this work reports on the growth, processing and characterization of blue, green and yellow LEDs (p-i-n structures) based on ordered NCs with much longer InGaN active regions (between $250 \mathrm{~nm}$ and $500 \mathrm{~nm}$ ). The results obtained point to this specific geometry as a potential alternative to the use of embedded InGaN QDisks, as well as to standard planar geometries using QWs, for its application for LEDs. In addition, the fact that thick InGaN sections (above $250 \mathrm{~nm}$ ) embedded on p-i-n ordered NCs can be grown without traces of extended defects, as reported in this work, which is in contrast to the typical results for planar structures, makes this design very appealing for solar cells as well $[21,22]$.

\section{Experimental details}

All samples were grown by SAG on commercial GaN(0001)/ sapphire templates in a RIBER Compact 21 PA-MBE system. The impinging molecular fluxes were calibrated in (0001) $\mathrm{GaN}$ (for Ga and $\mathrm{N}$ ) and (0001) InN (for In) growth rate units $(\mathrm{nm} / \mathrm{min})$ [23]. Prior to the growth, the templates were covered by a thin Ti mask with a compact hexagonal lattice of nanoholes made by colloidal lithography. Details of this procedure can be found elsewhere [24]. The nanohole lattice had a period ( $\mathrm{P}$, distance between centers of closest holes) of $280 \mathrm{~nm}$, and diameter around $150 \mathrm{~nm}$. Determined by the mask design the $\mathrm{NCs}$ density was $\sim 1.15 / \mathrm{P}^{2} \sim 1.5 \mathrm{E} 9 \mathrm{~cm}^{-2}$. The initial filling factor, defined as the ratio between the NCs footprint and the wafer area, was 0.26 . However, during the growth of the InGaN/GaN:Mg nanocolumnar sections the NC diameter increased due to the effects of $\mathrm{In}$ and $\mathrm{Mg}$ on the radial growth rate [25, 26], as it can be seen in figures 1 (b) and (c), causing the filling factor to increase up to 0.72 .

Two samples (A1, A2) of NCs with stacked GaN:Mg/ InGaN/GaN:Si sections (p-i-n structures) were grown following a three-step process: (i) 2 h 30 min growth of GaN:Si $\mathrm{NCs}$ at $860^{\circ} \mathrm{C}$ (Ga-flux, $\Phi_{\mathrm{Ga}}=16 \mathrm{~nm} \mathrm{~min}^{-1}$ and $\mathrm{N}$-flux, $\Phi_{\mathrm{N}}=$ of $5 \mathrm{~nm} \mathrm{~min}^{-1}$ ) with a Si cell temperature of $950^{\circ} \mathrm{C}$; (ii) growth of an InGaN section at $650^{\circ} \mathrm{C}\left(\Phi_{\mathrm{Ga}}=2.3 \mathrm{~nm} \mathrm{~min}^{-1}\right.$, $\Phi_{\text {In }}=6.4 \mathrm{~nm} \mathrm{~min}^{-1}$, and $\Phi_{\mathrm{N}}=12 \mathrm{~nm} \mathrm{~min}^{-1}$ ) during $1 \mathrm{~h}$ in sample A1 and $30 \mathrm{~min}$ in sample A2 (thicknesses of $500 \mathrm{~nm}$ and $250 \mathrm{~nm}$ respectively); and (iii) $30 \mathrm{~min}$ growth of $\mathrm{GaN}: \mathrm{Mg}$ at $600{ }^{\circ} \mathrm{C}\left(\Phi_{\mathrm{Ga}}=10 \mathrm{~nm} \mathrm{~min}^{-1}\right.$ and $\left.\Phi_{\mathrm{N}}=7 \mathrm{~nm} \mathrm{~min}^{-1}\right)$ with a $\mathrm{Mg}$ cell temperature of $365^{\circ} \mathrm{C}$. Although the same growth conditions were used for the InGaN growth in both samples, LEDs emitting from blue up to yellow could be fabricated. This was caused by a temperature gradient of around $20^{\circ} \mathrm{C}$ along the wafer during the MBE growth (highest temperature at the edge of the wafer), leading to a varying In incorporation from the wafer center (higher In\%) to the edge (lower In\%).

Three different devices were processed from these samples: i) D1 emitting in the blue from sample A1; ii) D2 emitting in the green, and iii) D3 emitting in yellow, both originating from sample A2. According to the wavelengths, the estimated In contents [27] are $18.6 \%, 27 \%$ and $33.7 \%$ for D1, D2 and D3, respectively. The devices have a circular shape (defined by the mesa etching) of $100 \mu \mathrm{m}$ diameter. The n-type contact was a conventional Ti/Al/Ti/Au (20/200/40/ $55 \mathrm{~nm}$ ) electrode deposited by electron beam evaporation on the GaN template. On top of the NCs, a Ni/Au $(20 / 500 \mathrm{~nm}) \mathrm{p}-$ type electrode was deposited by thermal evaporation, using $500 \mathrm{~nm}$ of $\mathrm{Au}$ to ensure proper current spreading. Thermal evaporation was carried out under an incidence angle of around $30^{\circ}$ and sample rotation. The small distance between NCs (maximum filling factor of 0.72 ) facilitates the metallization of the top contact by direct evaporation, without using filling material between NCs. The annealing conditions for both ohmic contacts were $5 \mathrm{~min}$ at $450^{\circ} \mathrm{C}$ under $\mathrm{H}_{2}: \mathrm{N}_{2}$ atmosphere. The light was collected through the sapphire substrate, which leads to a strong light intensity reduction to 1 or $2 \%$ of its original value due to the GaN/sapphire and sapphire/air interfaces [19].

The nanostructures and devices were characterized by scanning electron microscopy (SEM), transmission electron microscopy (TEM) and scanning transmission electron microscopy (STEM). High resolution TEM and atomic resolution characterization was performed on a JEOL JEMARM200cF electron microscope (cold emission gun) operating at $200 \mathrm{kV}$ provided with a spherical aberration corrector in probe (current density $\sim 1.4 \times 10^{-9} \mathrm{~A}$ and probe size $\sim 0.08 \mathrm{~nm}$ ). Solid semi-angles between $11-22 \mathrm{mrad}$ were used for the acquisition of annular bright field (ABF) images [28] using an acquisition time of $38 \mathrm{~s}$ per frame. In addition the nanostructures were characterized using I-V measurements, and room temperature (RT) pulsed electroluminescence (EL) with a $1 \%$ duty cycle and $1 \mu$ s pulses.

\section{Results and discussion}

The low magnification image of a representative NC projected onto the (010) plane is displayed in figure 1(c). NCs have a wurtzite-type crystal structure which is directly observed in the atomically resolved ABF-STEM image shown in figure $1(\mathrm{~d})$, revealing the disposition of the Ga and $\mathrm{N}$ atomic columns. No structural defects are detected in the $\mathrm{GaN}: \mathrm{Si} / \mathrm{InGaN}$ sections (zone $\mathrm{A}$ in figure 1(c)), but at the $\mathrm{NCs}$ ' top (zone $\mathrm{B}$ in figure 1(c)) the formation of twin 

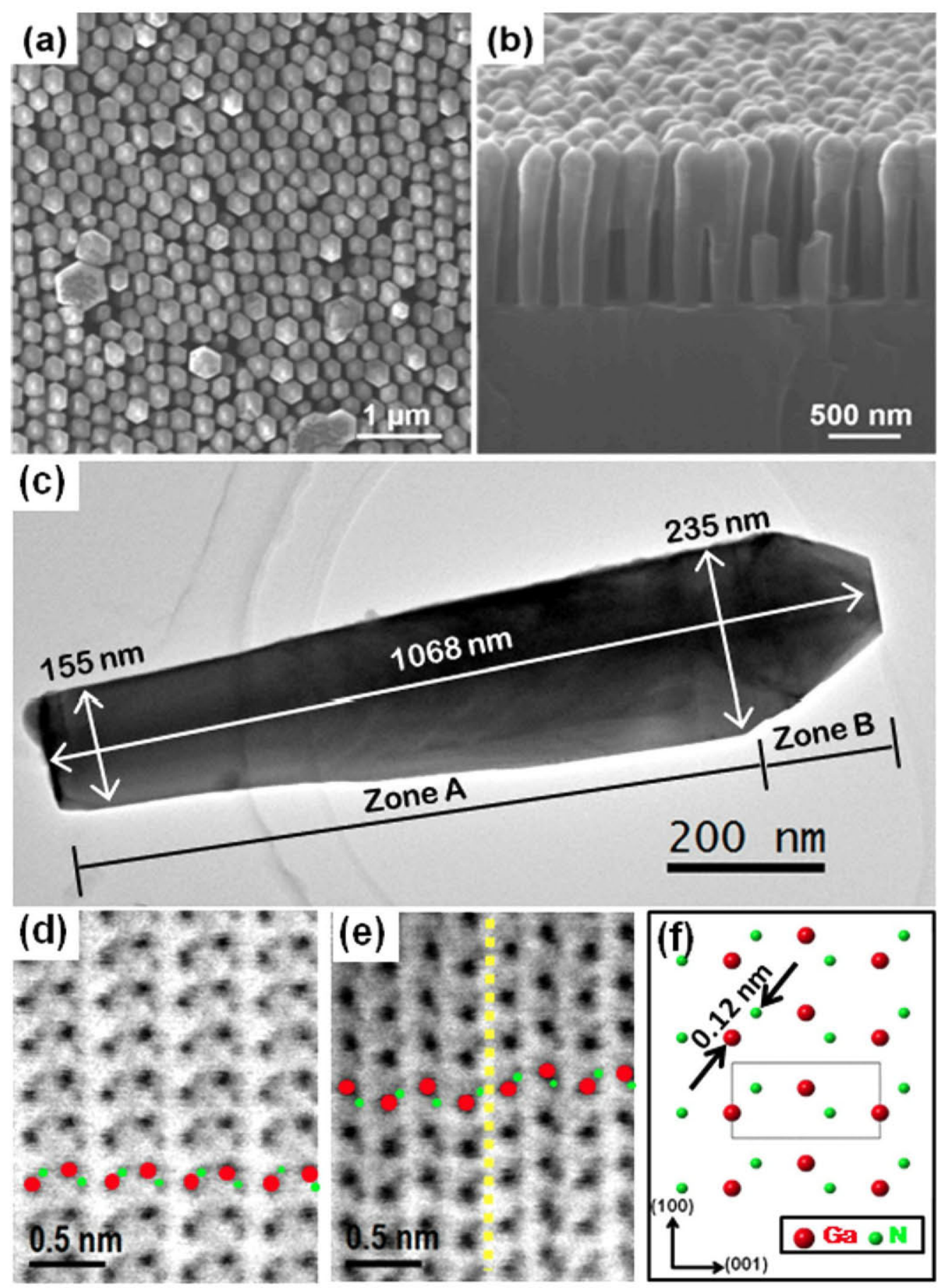

Figure 1. SEM pictures of sample A1: (a) top view, and (b) bird's-eye view; (c) low magnification TEM image of a single NC of sample A1; atomically-resolved ABF-STEM images of (d) GaN:Si/InGaN (zone A); and (e) GaN:Mg (zone B) highlighted sections (sample A1). (f) Schematic representation of $\mathrm{Ga}-$ and $\mathrm{N}$-atoms position in wurtzite structure.

boundaries create one monolayer of a blende-type structure (dashed line in figure 1(e)). The appearance of these extended structural defects is most likely caused by the low temperature growth of the upper p-type GaN section. As no consecutive twins are observed, the formation of an extended blende-type structure at the $\mathrm{NCs}^{\prime}$ top can be ruled out.

Although the current-voltage (I-V) characteristics of the devices show a non-negligible leakage current, a rectifying behaviour with a reasonable turn-on voltage is found, as shown in figure 2(b). Since no filling material was used to isolate/passivate the NCs, the top ohmic contact could partially short-circuit the $\mathrm{p}$ - and $\mathrm{n}$-sides, provoking the observed leakage. Remarkably, no clear dependence between emission wavelength and turn-on voltage was found. The reason for this is unclear at this point, and is currently under investigation. A possibility may relate to the In distribution inside the NCs, e.g. in device D1, emitting in the blue range, regions of higher In-content exist that 

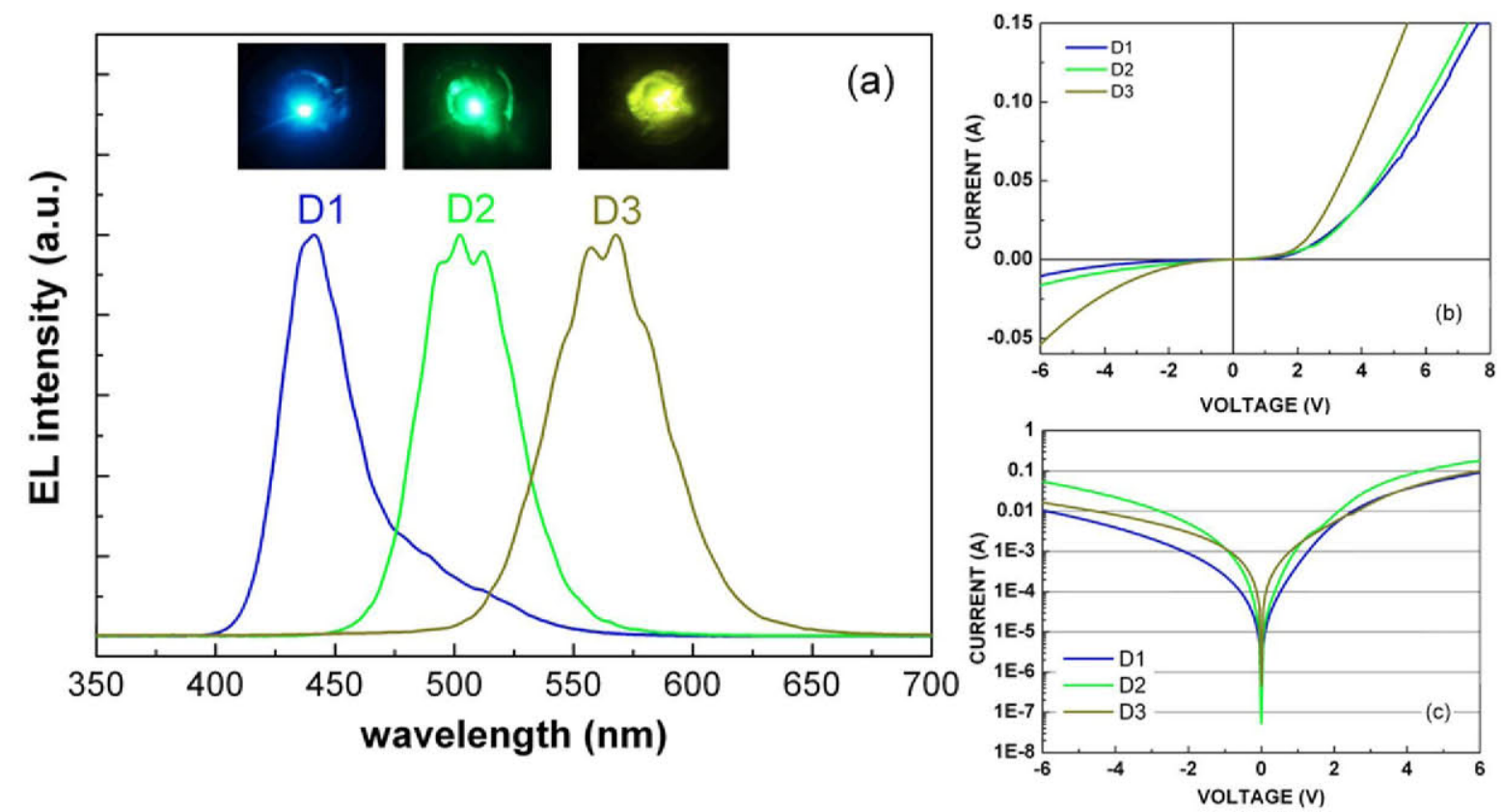

Figure 2. (a) Normalized RT-EL spectra of the three devices, at the maximum injected current (150 mA, nominal). The inset shows photographs of the light emission during the EL measurements. (b) I-V characteristic of the devices, and (c) the same I-V characteristics on semi-log plot.

dominate emission at lower injection currents, as will be discussed later on.

RT-EL measurements were performed from $10 \mathrm{~mA}$ (127 $\mathrm{A} \mathrm{cm}^{-2}$, considering the area of the circular device) to $150 \mathrm{~mA}\left(1910 \mathrm{~A} \mathrm{~cm}^{-2}\right)$ in pulsed mode to avoid self heating effects, with a $1 \%$ duty cycle and pulses of $1 \mu \mathrm{s}$. Taking into account the observed leakage currents for the three different devices, the actual injected currents can be estimated to be around $1700 \mathrm{~A} \mathrm{~cm}^{-2}, 1439 \mathrm{~A} \mathrm{~cm}^{-2}$ and $1600 \mathrm{~A} \mathrm{~cm}^{-2}$ for devices D1, D2 and D3, respectively. In addition it has to be noted that based on the calculation in reference [29], unpassivated NCs of the given diameter of $230 \mathrm{~nm}$ (figure 1(c)) would have an injection efficiency of only about $10 \%$. With that in mind the effectively injected current densities would be $170 \mathrm{~A} \mathrm{~cm}^{-2}, 144 \mathrm{~A} \mathrm{~cm}^{-2}$ and $160 \mathrm{~A} \mathrm{~cm}^{-2}$, which is still far beyond the normal operation range of commercial LEDs. On the other hand, since the NCs do not cover the whole circular device area (filling factor $<1$ ), the actual current densities would be higher than the ones calculated considering the device circular area. Normalized RT-EL spectra at the maximum injected current $(150 \mathrm{~mA}$ nominal) are shown in figure 2(a) for the three devices. At this maximum injected current, EL spectra peak at $441 \mathrm{~nm}$ (D1), $502 \mathrm{~nm}$ (D2) and $568 \mathrm{~nm}$ (D3). In the case of the blue device (D1), a pronounced tail clearly extends into longer wavelengths. This issue will be discussed later.

Assuming that extraction efficiency is not dependent on excitation, the $\mathrm{EQE}$ can be considered proportional to the ratio between the integrated EL intensity (L) and the current density $(\mathbf{J})$. No absolute values of $\mathrm{EQE}$ can be provided due to setup limitations.

Figure 3 shows the L/J ratio as a function of the (nominal) current density (as explained before, the actual value for

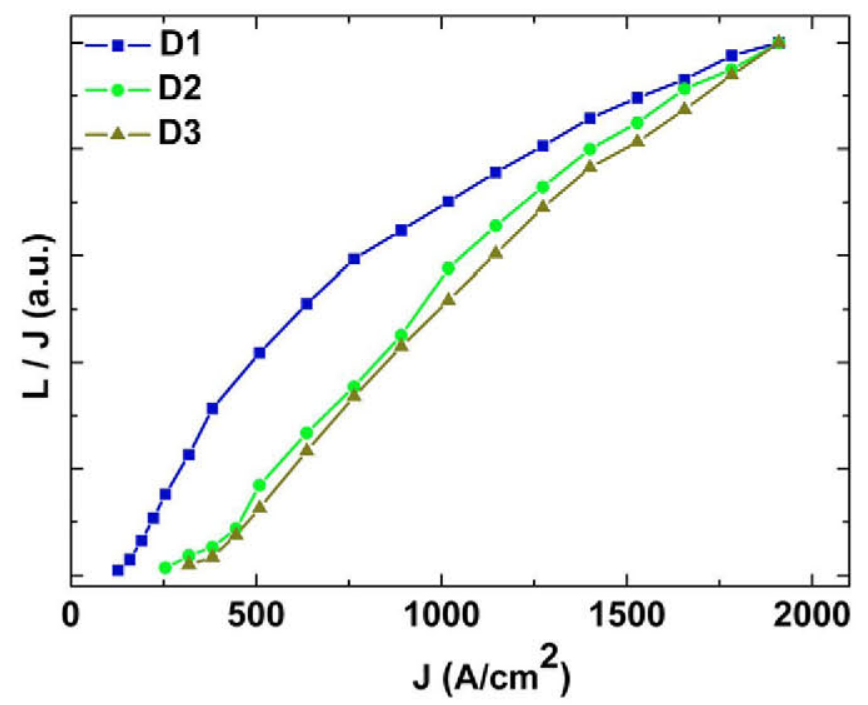

Figure 3. L/J versus J dependence for devices D1, D2 and D3.

the maximum current density differs from the nominal value) for the three devices, where no evidence of efficiency droop is observed. In standard planar LEDs, EQE reaches its maximum value at current densities around $10 \mathrm{~A} \mathrm{~cm}^{-2}$ or smaller, with efficiency droop at higher injection densities. Only in some approaches, most of them involving low-defective substrates, it is possible to shift the maximum $\mathrm{EQE}$ value to higher currents [4-6]. On the other hand, in the case of devices based on self-assembled NCs, high current densities are reached with virtually no efficiency droop, as in reference [12], with current densities up to $2200 \mathrm{~A} \mathrm{~cm}^{-2}$.

According to the $A B C$ model [1] the $\mathrm{EQE}$ can be modeled taking into account three parameters: i) the 
Shockley-Read-Hall (SRH) coefficient, named A, related to nonradiative recombination at crystal defects; ii) the radiative coefficient, named B; and iii) the nonradiative Auger coefficient, named $\mathrm{C}$. Following this model, the maximum efficiency is reached when the carrier density $(\mathrm{n})$ equals $(\mathrm{A} / \mathrm{C})^{0.5}$. The highly increased active volume of the devices presented here compared to standard ones based on QWs, leads to a lower carrier density for a given injection current density. Thus the EQE is expected to peak at higher current densities than in standard QW based devices. Figure 3 may indicate that the EL experiments were carried out at low enough carrier density so that the EQE peak is not reached. On the other hand it has to be noted that usually the fact that the EQE peak is not reached at high injection may also indicate a high defect related nonradiative recombination, although in the present case, it would be surprising given the absence of extended defects, as shown in figure 1 (point defects may still be present). However, considering the slow rise of $\mathrm{L} / \mathrm{I}$ values with increasing injection, and that the NCs are not passivated, a strong nonradiative surface recombination at the InGaN sidewalls may take place, which would lead to a reduction of the injection efficiency and thus of the internal quantum efficiency as shown in self-assembled nanocolumnar LEDs [29]. In that respect, no conclusive point can be made in terms of the absence of the droop effect.

The evolution of the EL peak wavelength with current density for the three devices is presented in figure 4(a), and the corresponding shifts $(\Delta \lambda)$ are indicated for devices D1 and D2. Device D3 (yellow) shows no wavelength shift, while device D2 (green) shows a blue-shift of $14 \mathrm{~nm}$ within the current density range between $254 \mathrm{~A} \mathrm{~cm}^{-2}$ and $891 \mathrm{~A} \mathrm{~cm}^{-2}$, followed by an almost constant value up to the maximum injected current. A similar behavior is found for device D1 (blue), which shows the largest blue shift (around $18 \mathrm{~nm}$ between $159 \mathrm{~A} \mathrm{~cm}^{-2}$ and $509 \mathrm{~A} \mathrm{~cm}^{-2}$ ) among all three devices.

Figure 4(b) shows a general trend of line width reduction with current injection in all three devices, more pronounced for D1 (until a stable value is reached beyond $1000 \mathrm{~A} \mathrm{~cm}^{-2}$ ), followed by D3, and finally an almost negligible effect for D2. At the highest injection current $(150 \mathrm{~mA})$ the measured EL line widths are of $35 \mathrm{~nm}, 45 \mathrm{~nm}$ and $55 \mathrm{~nm}$ for the blue $(441 \mathrm{~nm})$, green $(502 \mathrm{~nm})$ and yellow $(568 \mathrm{~nm})$ devices (figure 4(c)). A similar trend has been reported for planar QW based LEDs [30,31], namely, $20 \mathrm{~nm}, 35 \mathrm{~nm}$ and $50 \mathrm{~nm}$ for blue, green and orange LEDs, and was explained in terms of increasing alloy disorder for higher In contents. The higher line width values measured in D1 to D3 LEDs can be attributed to: (i) slight variations of NCs diameters due to a less than perfectly regular colloidal mask and, consequently, of In content [15], thus broadening the EL spectrum (the effects on NC diameter by mask geometry dispersion can be seen in figure 1(a)); and (ii) In\% distribution within the InGaN section on each $\mathrm{NC}$ due to lattice pulling and $\mathrm{In}(\mathrm{Ga}) \mathrm{N}$ thermal decomposition [32, 33]. Remarkably, the EL spectral line widths of devices D1 to D3 are narrower than those reported for LEDs using ordered NCs with InGaN QDisc
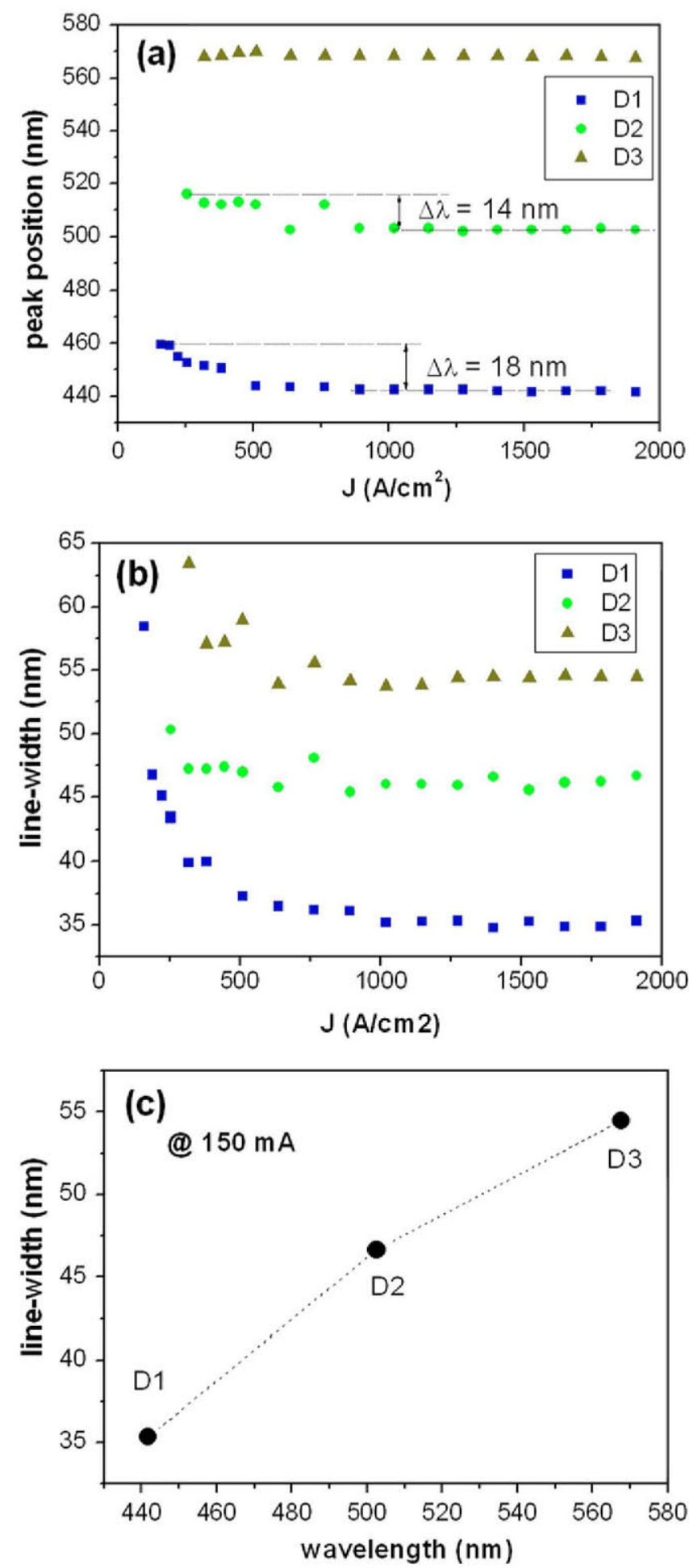

Figure 4. (a) EL peak wavelength dependence on current density indicating the peak shifts for devices D1 and D2; (b) EL spectra line width evolution with current density for devices D1, D2 and D3; and (c) EL spectra line widths for the three devices, at the maximum injected current $(150 \mathrm{~mA})$.

active regions $(67 \mathrm{~nm}, 96 \mathrm{~nm}, 54 \mathrm{~nm}$ at a peak wavelengths of $544 \mathrm{~nm}, 583 \mathrm{~nm}$ and $597 \mathrm{~nm}$ respectively) [18].

The typical blue shift and line width narrowing observed in InGaN QWs and QDs upon increasing excitation has generally been explained by screening of the internal electric 
field $[34,35]$. The same trend is found in the devices analyzed in this work (figures 4(a) and (b)) though in this case the most reasonable explanation relies on the presence of localized states within the InGaN sections. In the present work, it can be speculated that different distributions and depths of localized states may be present in the three devices D1, D2 and D3. In device D1 (blue LED) a low-density distribution of localized states is assumed to be present close to the conduction band (CB) bottom, whereas a distribution of localized states with higher density and depth, but much wider spread in energy as to merge into the $\mathrm{CB}$, may be present in devices D2 and D3 due to a higher In-content. In addition, it has to be taken into account that the residual n-type carrier density increases with increasing In $\%$ [36] leading to different positions of the Fermi level with respect to the $\mathrm{CB}$ edge in the three devices. Having in mind both the differences in localization and Fermi level position, the energy blue shift and the reduction of FWHM with increasing injection current can partially be understood.

In the blue LED (device D1) the Fermi level position is assumed to be slightly above the localized states. At low injection current, emission from localized states is assumed to dominate (low energy peak). With increasing injection current, the Fermi level would shift into the $\mathrm{CB}$ and consequently the EL emission would be dominated by the CB states (high energy peak). Due to the much higher density of states (DOS) in the $\mathrm{CB}$, an overall reduction of the line width with increasing injection current would be observed. This interpretation is backed up by the data from the EL spectrum in figure 5(a), where two resolved EL peaks with similar intensity are observed at low current injection; one at higher energy (emission from the $\mathrm{CB}$ ) and a second one at lower energy (emission from localized states). When increasing the current injection, the higher energy peak becomes dominant (much higher CB DOS) with a concomitant narrowing of the emission peak. At this point it has to be noted that no significant shift of the Fermi level is assumed to take place once the $\mathrm{CB}$ is reached. This assumption is reasonable when taking into account the efficient recombination of carriers as well as the high effective masses (flat band) in nitrides.

In the green (D2) and yellow (D3) devices (EL spectra shown in figures $5(\mathrm{~b})$ and (c)) the EL peak width at low injection current is assumed to reflect the energy spread of all the localized states (merging into the $\mathrm{CB}$ ), which all add to the emission due to the Fermi level position well above them (which is due to the higher In content). An increase of the injection current would start 'filling up' the localized states (which have a much higher DOS compared to devices D1), as well as CB states, once all localized states are filled, which would again cause a blue shift as well as reduction of the line width as in the case of the blue device, although much less severe due to the higher DOS of the localized states in devices D2 and D3.

However, it cannot be ruled out that the blue shift, as well as line-width reduction, is also related to different filling states of the localized states inside the InGaN volume. Since the center of the InGaN volume can be assumed to have a higher In content than the verges [37], at a low injection
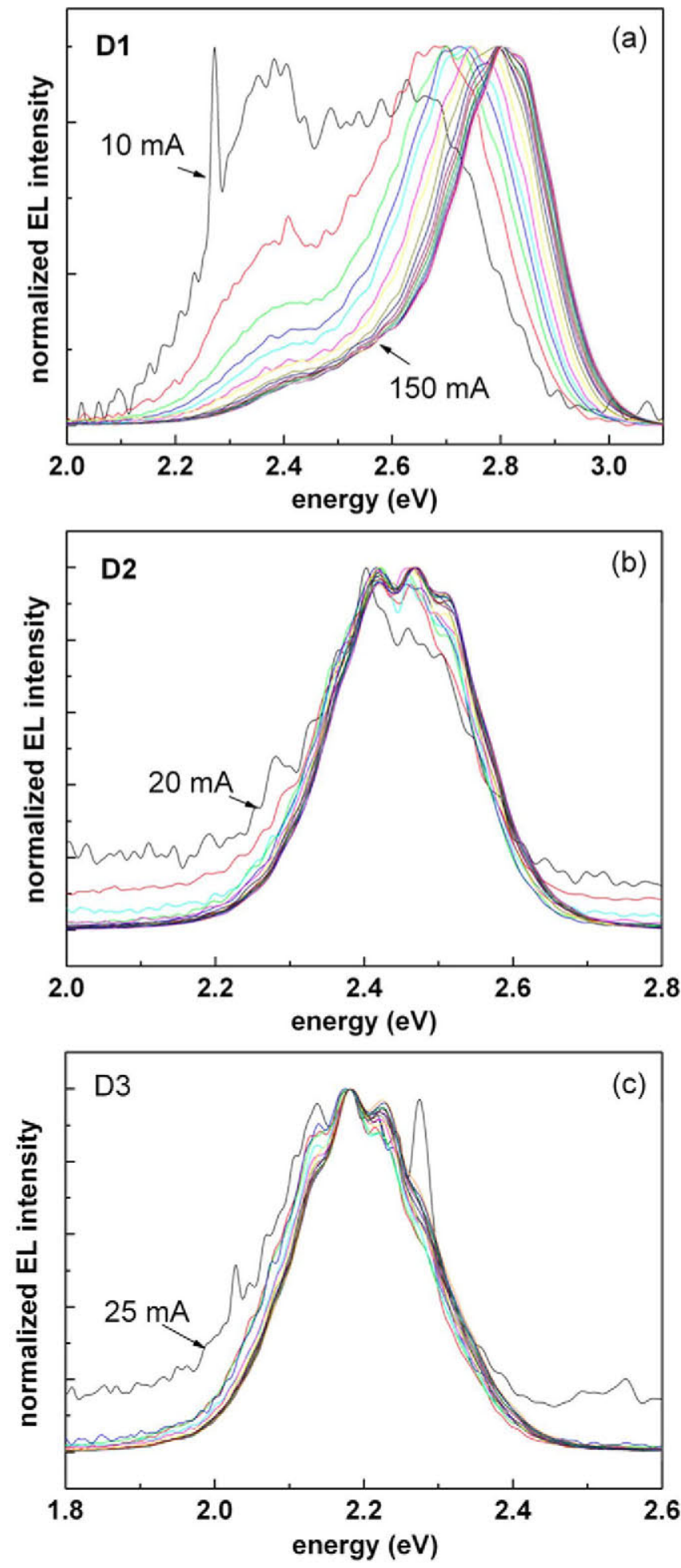

Figure 5. (a) EL spectra of device D1, from low injected current $(10 \mathrm{~mA})$ to high injected current $(150 \mathrm{~mA})$. At low injection, emission is formed by two peaks of similar intensities, while at high injection, the low energy peak is attenuated. (b) and (c) are the EL spectra for devices D2 and D3, respectively.

current it is reasonable to assume that the carriers tend to fill the localized states towards the center of the InGaN volume, while on the verges of the central zone the filling will be lesser. Due to this inhomogeneous saturation degree, the 
emission would be rather broad. Upon increasing injection current, the central (saturated) recombination region tends to spread along the entire InGaN volume and eventually saturation of localized states extends over the entire InGaN volume, thus reducing the line width. This effect would be more pronounced for devices D2 and D3 due to the higher length of the InGaN segment.

\section{Conclusion}

In summary, blue $(441 \mathrm{~nm})$, green $(502 \mathrm{~nm})$ and yellow $(568 \mathrm{~nm}) \mathrm{LEDs}$, based on ordered NCs having long InGaN active regions $(250 \mathrm{~nm}$ to $500 \mathrm{~nm})$, were successfully achieved. The long InGaN segments were found to be defectfree and the only twin boundaries were found in the upper GaN:Mg region, most likely due to the low temperature used for the growth of this region. Pulsed EL measurement up to (nominally) $1900 \mathrm{~A} \mathrm{~cm}^{-2}$ did not show droop or saturation of the efficiency, while the emission wavelength experiences only a small blue shift, almost negligible for the yellow device $(568 \mathrm{~nm})$. In addition, a narrowing of the emission line widths was found. Finally, the line width values measured are slightly higher than those reported for planar MQW structures, but in the range of, or better than, the values reported for ordered nanoLEDs using InGaN QDiscs. These nanoLEDs with embedded thick InGaN regions may have potential in lighting applications, since they combine the ordered NCs advantages (high homogeneity, quality, light extraction efficiency, etc) with the benefits of a thick InGaN active region (reduction of Auger recombination and current overflow mechanism) without crystal quality degradation.

\section{Acknowledgments}

We acknowledge partial financial support by the EU FP7 Contract GECCO 280694, the EU ITN RAINBOW PITNGA-2008-213238, and Ministerio de Ciencia e Innovación: MAT2011-26703.

\section{References}

[1] Piprek J 2010 Phys. Status Solidi A 207 2217-25

[2] Kioupakis E, Rinke P, Delaney K T and Van de Walle C 2011 Appl. Phys. Lett. 98161107

[3] Vampola K J, Iza M, Keller S, DenBaars S P and Nakamura S 2009 Appl. Phys. Lett. 94061116

[4] Gardner N F, Müller G O, Shen Y C, Chen G, Watanabe S, Götz W and Krames M R 2007 Appl. Phys. Lett. 91243506

[5] Maier M, Köhler K, Kunzer M, Pletschen W and Wagner J 2009 Appl. Phys. Lett. 94041103

[6] Liu Z, Wei T, Guo E, Yi X, Wang L, Wang J, Wang G, Shi Y, Ferguson I and Li J 2011 Appl. Phys. Lett. 99091104

[7] Wang C H et al 2010 Appl. Phys. Lett. 97261103
[8] Kikuchi A, Kawai M, Tada M and Kishino K 2004 Jpn. J. of Appl. Phys. 43 L1524

[9] Kim H-M, Cho Y-H, Lee H, Kim S I, Ryu S R, Kim D Y, Kang T W and Chung K S 2004 Nano Lett. 41059

[10] Guo W, Zhang M, Banerjee A and Bhattacharya P 2010 Nano Lett. 103355

[11] Nguyen H P T, Zhang S, Cui K, Han X, Fathololoumi S, Couillard M, Botton G A and Mi Z 2011 Nano Lett. 111919

[12] Nguyen H P T, Cui K, Zhang S, Djavid M, Korinek A, Botton G A and Mi Z 2012 Nano Lett. 121317

[13] Henneghien A-L, Tourbot G, Daudin B, Lartigue O, Désières Y and Gérard J-M 2011 Optics Express 19527

[14] Bavencove A L, Tourbot G, Garcia J, Désières Y, Gilet P, Levy F, Andre B, Gayral B, Daudin B and Dang L S 2011 Nanotechnology 22345705

[15] Sekiguchi H, Kishino K and Kikuchi A 2010 Appl. Phys. Lett. 96231104

[16] Albert S, Bengoechea-Encabo A, Kong X, Sanchez-Garcia M A, Calleja E and Trampert A 2013 Appl. Phys. Lett. 102181103

[17] Albert S, Bengoechea-Encabo A, Sanchez-Garcia M A, Kong X, Trampert A and Calleja E 2013 Nanotechnology 24 175303

[18] Kishino K, Nagashima K and Yamano K 2013 Appl. Phys. Express 6012101

[19] Vadivelu R, Igawa Y and Kishino K 2013 Jpn. J. Appl. Phys. 52 08JE18

[20] Kishino K, Kamimura J and Kamiyama K 2012 Appl. Phys. Express 5031001

[21] Feng S-W, Lai C-M, Chen C-H, Sun W-C and Tu L-W 2010 J. Appl. Phys. 108093118

[22] Golam S A T M and Myers R C 2012 Appl. Phys. Lett. 101 143905

[23] Heying B, Averbeck R, Chen L F, Haus E, Riechert H and Speck J S 2000 J. Appl. Phys. 881855

[24] Bengoechea-Encabo A et al 2012 J. Crys. Grow. 353 1-4

[25] Albert S, Bengoechea-Encabo A, Lefebvre P, Sanchez-Garcia M A, Calleja E, Jahn U and Trampert A 2011 Appl. Phys. Lett. 99131108

[26] Furtmayr F, Vielemeyer M, Stutzmann M, Arbiol J, Estradé S, Peirò F, Morante J R and Eickhoff M $2008 \mathrm{~J}$. Appl. Phys. 104034309

[27] Wu J and Walukiewicz W 2003 Superlattices \& Microstructures 3463

[28] Ishikawa R, Okunishi E, Sawada H, Kondo Y, Hosokawa F and Abe E 2011 Nat. Materials 10 278-81

[29] Nguyen H P T, Zhang S, Connie A T, Kibria M G, Wang Q, Shih I and Mi Z 2013 Nano Lett. 135437

[30] Mukai T, Yamada M and Nakamura S 1999 Jpn. J. Appl. Phys. 383976

[31] Enya Y et al 2009 Appl. Phys. Express 2082101

[32] Kong X, Albert S, Bengoechea-Encabo A, Sanchez-Garcia M A, Calleja E and Trampert A 2012 Nanotechnology 23485701

[33] Albert S, Bengoechea-Encabo A, Sanchez-Garcia M A, Calleja E and Jahn U 2013 J. Appl. Phys. 113114306

[34] Wang T, Nakagawa D, Wang J, Sugahara T and Sakai S 1998 Appl. Phys. Lett. 733571

[35] Park I-K, Kwon M-K, Cho C-Y, Kim J-Y, Cho C-H and Park S-J 2008 Appl. Phys. Lett. 92253105

[36] Li S X, Yu K M, Wu J, Jones R E, Walukiewicz W, Ager J W III, Shan W, Haller E E, Lu H and Schaff W J 2005 Phys. Rev. B 71161201

[37] Tourbot G, Bougerol C, Grenier A, Den Hertog M, Sam-Giao D, Cooper D, Gilet P, Gayral B and Daudin B 2011 Nanotechnology 22075601 